\title{
Alternde Musiker haben jüngere Ohren
}

\begin{abstract}
Mit zunehmendem Alter wird es immer schwerer, Sprache zu verstehen, wenn sie von Hintergrundgeräuschen überlagert wird. Schuld daran ist nicht ausschließlich das nachlassende Hörvermögen. Auch die Hirnleistung lässt nach, und es wird immer schwieriger, Informationen aufzunehmen und zu verarbeiten.
\end{abstract}

— Musiker haben es da besser. Nach einer Studie von Wissenschaftlern der Northwestern University in Evanston (USA) besitzen ältere Musiker nicht nur ein besseres Gedächtnis, sondern sie können auch unter schwierigen Bedingungen besser hören und verstehen.

In einer kleinen Studie mit 18 Musikern und 19 Nicht-Musikern im Alter von 45 bis 65 Jahren konnte in zahlreichen Tests gezeigt werden, dass $\mathrm{Mu}$ sik die Fähigkeit fördert, Klangmuster aus Lärm herauszufiltern und zu erkennen. So konnte Sprache trotz lauter Geräuschkulisse besser verstanden werden. Musiker, die seit ihrem neun-

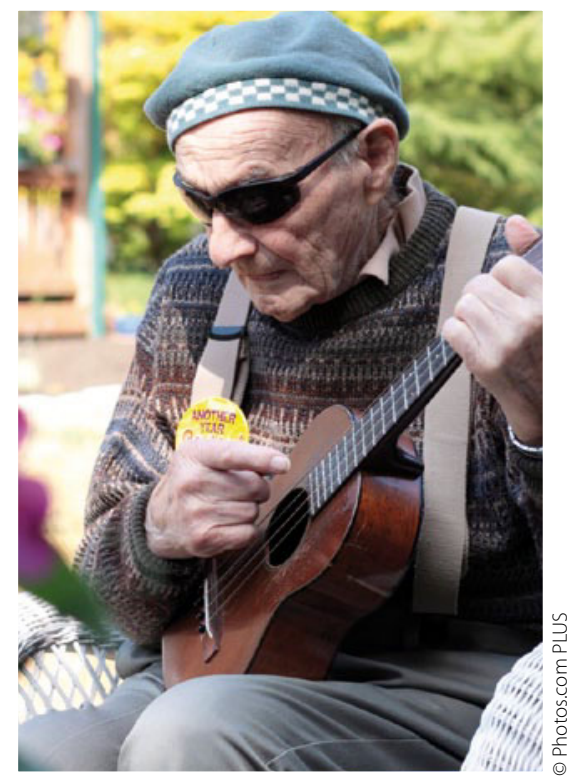

Wer lebenslang musiziert, kann auch im Alter Sprache gut erkennen.

Kommentar

Durch Musizieren lässt sich das altersbedingte Nachlassen des Hörens reduzieren. Die neuronalen Verbesserungen bei musikalisch Trainierten sind nach Meinung der Autoren nicht einfach ein Lautstärkereglereffekt, sondern ein Feintuning des zentralen Nervensystems durch das Musizieren, wodurch auch das Spracherkennungsvermögen verbessert wird.

K. MALBERG -

ten Lebensjahr beständig ein Instrument spielten, schnitten in allen Bereichen der Gedächtnisleistungen, des Hörens und des Sehens besser ab. Hingegen waren Leistungen, die mit dem visuellen Arbeitsgedächtnis zusammenhingen, in beiden Gruppen gleich.

\section{Wenn Propofol schmerzhaft ist}

\section{Will man den Schmerz bei der Propofolinjektion vermeiden, wählt man am besten eine Kubitalvene.}

- Das kurz wirkende Narkotikum Propofol wird seit einigen Jahren bei Millionen von Patienten angewendet, die sich kleinen Operationen oder einer invasiven Diagnostik, insbesondere der Endoskopie unterziehen. Propofol erfreut sich wegen der kurzen Wirkdauer, der guten Titrierbarkeit und des günstigen Nebenwirkungsspektrums großer Beliebtheit. Allerdings berichten drei von fünf Patienten über Schmerzen an der Injektionsstelle.

In einem systematischen Review und einer Metaanalyse durch Recherche in einschlägigen medizinischen Datenbanken konnten 177 randomisierte und kontrollierte Studien mit zusammen 25260 Patienten gefunden werden, die dieses Problem behandeln. Das Gesamtrisiko für eine schmerzhafte Empfindung bei der Propofolinjektion betrug etwa $60 \%$. Als effektivste Maßnahme zur Vermeidung der Schmerzen erwies sich die Injektion in eine Kubitalvene anstelle einer Handrückenvene. Durchaus noch wirksam waren eine Vorbehandlung mit Lidocain in die ungestaute Vene, die vorherige Injektion von Opioiden, Ketamin in einer Dosis zwischen 5 und 75 mg oder NSAR.

\section{Kommentar}

Man darf annehmen, dass die Injektion in eine Kubitalvene den Kontakt der Substanz mit der Venenwand vermeidet oder zumindest verkürzt. Möglicherweise wird auch Propofol durch einen höheren venösen Rückstrom rascher verdünnt. Die Kubitalvene hat den Nachteil, dass der Zugang durch Abknicken des Arms leicht verlegt wird, doch kann das Risiko für einen Injektionsschmerz um 85\% gesenkt werden. Ist man auf eine Handvene angewiesen, so empfiehlt es sich, entweder eine kleine Dosis eines Opioids vor der Anästhesieeinleitung mit Propofol zu verabreichen oder Lidocain und Propofol zu mischen.

H. S. FÜESSL "

\footnotetext{
- L. Jalota et al.

(Korresp.: C.C. Apfel, apfelc@anethesia.ucfs. edu) Prevention of pain on injection of propofol: systemic review and meta-analysis. Brit. med. J. 342 (2011) d1110
} 